\title{
Necesidad de cambios organizativos en el Sistema Nacional de Salud para la atención del ictus
}

\author{
Need of organizational changes in the National Health System \\ to give attention to stroke
}

\author{
Otman Fernández Concepción';Miguel Ángel Buergo Zuaznabar' \\ 'Especialista de II Grado en Neurología. Instituto de Neurología y Neurocirugía. La \\ Habana, Cuba.
}

\begin{abstract}
RESUMEN
La atención al paciente con accidente cerebrovascular (ictus) se ha revolucionado en las últimas dos décadas debido a que la investigación científica ha producido un conjunto de resultados que apoyan la eficacia de dos estrategias para el tratamiento de urgencia: la hospitalización de pacientes en unidades de ictus y el tratamiento trombolítico cerebral. Tanto la primera como la segunda, reducen de forma significativa las muertes y la discapacidad, y con ello el costo derivado de la atención postictus. Las unidades de ictus garantizan una atención dedicada y requiere de cambios en la infraestructura de los hospitales. La trombolisis cerebral, para que sea efectiva es dependiente del tiempo, por lo se requiere de un sistema organizado desde la atención prehospitalaria hasta el ingreso del paciente. Los sistemas de salud en la mayoría de los países desarrollados, y gran parte de otros en vía de desarrollo, han introducido modificaciones sustanciales en ese sentido; en tanto, las condiciones actuales de atención a quienes sufren un ictus en Cuba, han variado poco en relación con la situación que existía en la década de los años 80 y principios de los 90 . En este artículo se revisan los resultados que apoyan un grupo de recomendaciones a las autoridades de salud, para introducir cambios en el sistema de atención a estos pacientes.
\end{abstract}

Palabras clave: Accidente cerebrovascular, ictus, sistemas de salud, trombolisis terapéutica, tratamiento de urgencia. 


\begin{abstract}
Stroke patient care has been revolutionized in the last two decades since scientific research has produced a series of results that support the efficacy of two emergency treatment strategies: admission of patients to stroke units and brain thrombolysis treatment. Both strategies significantly reduce death and disability and thus, the cost of post-stroke care. Stroke units assure customized care, which requires changes in hospital infrastructure. Brain thrombolysis depends on time to be effective, so an organized system from pre-hospital care to patient hospitalization is needed. Health systems in most of the developed countries and in a large number of developing nations have introduced substantial changes in this aspect; however, the present conditions of care to those patients suffering from stoke in Cuba have varied a little since the $80^{\prime} \mathrm{s}$ and the beginning of the $90^{\prime} \mathrm{s}$. This article reviewed the results supporting several recommendations for the health authorities, with a view to making changes in the care to the stroke patients.
\end{abstract}

Key words: Stroke, health systems, therapeutic thrombolysis, emergency treatment.

\title{
I NTRODUCCI ÓN
}

La década de los 90 y el inicio del siglo xxi han sido fructíferos en investigaciones sobre el ictus (accidente vascular cerebral); el nihilismo reinante en los 80 ha cedido paso ante un mejor conocimiento sobre sus causas, su fisiopatología y su patogenia, se conocen los factores de riesgo que explican el $75 \%$ de la ocurrencia, se dispone de métodos eficaces para el manejo y tratamiento en la fase aguda, existen medidas eficaces para la prevención secundaria, y se cuenta con mejores estrategias para la rehabilitación.

La atención al ictus isquémico agudo hoy, requiere un sistema organizado desde la atención prehospitalaria hasta el egreso del paciente que asegure la continuidad de la rehabilitación. Aunque esto constituye un reto para los sistemas de salud, la mayoría de los países desarrollados y muchos en vías de desarrollo, han introducido cambios sustanciales en la organización de la atención de estos enfermos. Estas modificaciones se basan en evidencias contundentes sobre dos estrategias de tratamiento que reducen marcadamente las muertes y la discapacidad, y con ello el costo derivado de la atención post-ictus. Estas son: la hospitalización de pacientes en unidades de ictus (UI), y el tratamiento trombolítico cerebral (TC).

Es propósito de este trabajo revisar los hechos que sustentan la necesidad de cambios organizativos para la atención de estos pacientes, y realizar recomendaciones a las autoridades de salud sobre esta base. 


\section{EL PROBLEMA}

Al analizar la situación de la atención médica al paciente con ictus que existe hoy en Cuba, se observa lo siguiente:

- La población desconoce la urgencia del ictus; la educación a la población en relación con el ictus ha sido insuficiente y no ha tenido impacto en el conocimiento sobre el tema.

- El personal médico desconoce la urgencia del ictus como una emergencia similar al infarto agudo del miocardio y el politraumatismo.

- La atención médica a estos pacientes descansa en personal médico y de enfermería sin adiestramiento o dedicación al manejo de estos enfermos. • El sistema de traslado por ambulancias es ineficiente y no tiene al ictus como prioridad.

- Los pacientes con ictus se hospitalizan en salas abiertas de medicina, en terapias intermedias generales, o en terapias intensivas generales (los menos).

- Alta frecuencia de indicaciones de medicamentos que no han mostrado eficacia para la fase aguda, tales como pentoxifilina, piracetam, nimodipino y vitaminas antioxidantes, terapias potencialmente iatrogénicas como el uso rutinario de manitol, soluciones hipoosmolares (como la dextrosa $5 \%$ ), y medicamentos antihipertensivos que tienen igual riesgo al utilizarse de forma rutinaria; también el uso de métodos diagnósticos ineficaces como la punción lumbar.

- No existe disponibilidad de medicamentos de primera línea en todas las etapas del manejo del enfermo: no se cuenta con el trombolítico factor tisular activador del plasminógeno (rtPA) para el tratamiento específico del infarto cerebral, en escasos centros se realiza un manejo adecuado y urgente de pacientes con hemorragia subaracnoidea, y no se dispone de antiplaquetarios alternativos a la aspirina como el clopidogrel.

- Existe atraso tecnológico en medios diagnósticos accesibles y de equipamiento para el tratamiento: los equipos de neuroimagen no están en disposición de la urgencia para el ictus en la mayoría de los centros y no se dispone de equipos para el estudio vascular (equipos de Doppler transcraneal, angiógrafos).

Esto pudiera muy bien representar la situación de atención al ictus de la década de los años 80 y principios de los 90 , sin embargo, desde entonces se han producido investigaciones que aportan resultados suficientes que estimulan a un cambio en la forma de ver esta enfermedad y la atención a estos pacientes. A continuación, se revisan los resultados disponibles que apoyan dos estrategias para el manejo del ictus isquémico agudo, que han sido aprobadas por asociaciones científicas (internacionales y nacionales) dedicadas el ictus y autoridades de salud de la mayor parte de los países desarrollados y gran parte de los países en vías de desarrollo. Estas son: las unidades de ictus y la trombolisis cerebral.

\section{Sobre las unidades de ictus}

Según la OMS, la unidad de ictus (UI) es un dispositivo específico de cuidados agudos no intensivos para el tratamiento de pacientes con ictus, que ofrece el cuidado más efectivo al ictus agudo. El Grupo para el Estudio de Enfermedades Cerebrovasculares (GEECV) de la Sociedad Española de Neurología (SEN) la define de manera más completa:

"...una estructura geográficamente delimitada para el cuidado de pacientes con ictus, que tiene un personal entrenado, específicamente dedicado, coordinado por un neurólogo experto, con servicios diagnósticos disponibles durante las $24 \mathrm{~h}$ del día, y que dispone de protocolos escritos para el manejo de los pacientes basados en evidencias científicas". ${ }^{1}$ 
Los beneficios absolutos de la atención hospitalaria en UI son lo suficientemente grandes como para justificar una reorganización de los servicios. De acuerdo a una revisión sistemática de ensayos clínicos aleatorizados (ECAs) de la Colaboración Cochrane, donde se incluyeron 23 ensayos clínicos, la atención en UI mostró reducción en las muertes registradas en el seguimiento final al año (OR: 0,86; IC 95\%: 0,71-0,94), en el riesgo de muerte o institucionalización (OR: 0,80; IC 95\%: 0,71-0,90) y el de muerte o dependencia (OR: 0,78; IC 95\%:0,68-0,89). EI número necesario a tratar (NNT) para garantizar un resultado "favorable" es de 33 para supervivencia, 20 para alcanzar un grado de independencia y 20 para retornar al domicilio. ${ }^{2}$ Este y otros estudios demostraron que el beneficio persiste después de ajustar por edad, sexo y gravedad del déficit neurológico inicial y distintos subtipos de ictus, sin producir una duración mayor en la estadía hospitalaria. ${ }^{3,4}$

Una de las posibilidades que ofrece la UI es el monitoreo continuo de los indicadores fisiológicos; esto se asocia a menor mortalidad y 2,5 veces mayor probabilidad de tener una buena evolución al alta, y permitir la detección temprana de las complicaciones y su tratamiento rápido. ${ }^{5,6}$

Recientemente se publicó el Research Project on Stroke Services in Italy (PROSIT), estudio encargado por el Ministerio de Salud de Italia para definir la introducción de esta estrategia de forma generalizada en el país. Fue un estudio prospectivo que comparó la evolución de los pacientes atendidos en UI y aquellos hospitalizados en sala convencional de Neurología. Se incluyeron 11572 pacientes en las primeras 48 h de 260 hospitales italianos. Después de una media de 20 meses de seguimiento, se observó que los hospitalizados en UI tuvieron menor riesgo de morir o tener discapacidad (OR: 0,81; IC95\%; 0,72-0,91). ${ }^{7}$

Desde el punto de vista de los costos, los beneficios esperados por el ingreso de estos pacientes en UI justifican el mayor costo de la atención en la fase aguda, a expensas de una reducción de los costos de la atención postictus. ${ }^{8-10}$

Debido a que los beneficios obtenidos en los ECAs se desarrollan en condiciones "ideales", y difíciles de reproducir en la práctica, se ralizó una revisión sistemática sobre estudios observacionales de implementación de UI. Los investigadores identificaron 72 artículos, 25 de ellos fueron elegibles y 18 mostraron datos sobre casos fatales y mala evolución. El ingreso en UI redujo significativamente las muertes (OR: 0,79 ; IC95\%: 0,73-0,86) y la probabilidad de muertes más evolución pobre (OR: 0,87; IC95\%: 0,80-0,95) dentro del primer año después del ictus; de manera que los resultados son comparables a los obtenidos en ECAs. ${ }^{11}$

Con la remodelación de los hospitales en Cuba, muchos centros han creado hipotéticas "salas de ictus", al dedicar un espacio físico con un número de camas, habitualmente atendidos por el personal de las terapias intermedias; sin embargo, la UI es un concepto diferente. Los resultados antes expuestos están basadas en las características de las unidades de ictus incluidas en los ECAs.

En base a una extensa revisión de la literatura y el consenso de expertos, las UI deben cumplir una serie de requisitos mínimos que han sido definidos por la Brain Attack Collition (BAC), ${ }^{12}$ organismo especializado en recomendar estrategias para el manejo del ictus en los EE.UU.

Por ello, las UI y los centros en que se encuentran deben ser certificados por una comisión externa de expertos (Comité de certificación de centros y unidades para la atención de pacientes con ictus).$^{13}$ En Cuba esta comisión pudiera ser nombrada por organismos rectores o asesores como la Comisión Nacional de Enfermedades 
Cerebrovasculares y el Grupo Nacional de Neurología, rectorados por el Instituto de Neurología y Neurocirugía.

\section{Sobre la trombolisis}

El tratamiento trombolítico con rtPA es el único medicamento aprobado como primera línea para el tratamiento en la fase aguda del ictus isquémico por la mayoría de las asociaciones internacionales y nacionales. Fue aprobado por la Food and Drug Administration (FDA) de los EE.UU. para su uso en el ictus isquémico en 1996, basado fundamentalmente en el estudio del National Institute Neurological Disorder Stroke de los EE.UU. (NINDS, por sus siglas en inglés), en 1999 recibió licencia para su uso en Canadá, y en 2002 en la Unión Europea. Además, ha sido aprobado por autoridades de salud en otros 40 países. $^{1}$

El estudio rtPA del NINDS incluyó 624 pacientes, en dos etapas, que se presentaron dentro de las primeras $3 \mathrm{~h}$ del debut y fueron aleatorizados para asignarles tratamiento con rtPA endovenoso a dosis de $0,9 \mathrm{mg} / \mathrm{kg}$, o placebo. Aunque la mejoría neurológica entre ambos grupos no difirió después de $24 \mathrm{~h}$, el resultado a los 3 meses fue significativamente superior en el grupo tratado (OR: 2,11; IC $95 \%: 1,33-3,55)$.

La hemorragia intracerebral ( $\mathrm{HIC}$ ) ocurrió en el $6,4 \%$ de los pacientes tratados con rtPA comparado con el $0,6 \%$ en el grupo placebo. ${ }^{14} \mathrm{El}$ beneficio del tratamiento se mantuvo al año y sus resultados derivaron en una disminución significativa de los costos producidos por la atención post-ictus. ${ }^{15,16}$ Seguidamente a la aprobación por la FDA del tratamiento con rtPA, varios grupos informaron de la utilidad del tratamiento en varios estudios comunitarios, la mayoría de estos registraron tasas de HIC y de evolución favorable similares a los obtenidos por el estudio del NINDS.

El primer European Cooperative Acute Stroke Study (ECASS-I) comparó 1,1mg/kg de rtPA contra placebo en las primeras $6 \mathrm{~h}$ desde el debut; ambos grupos no difirieron significativamente. ${ }^{17}$ Otros dos ensayos clínicos, realizados en EE.UU., que utilizaron rtPA dentro de las primeras $3 \mathrm{~h}$ tampoco mostraron beneficio. ${ }^{18,19}$

Los investigadores del estudio NINDS demostraron una interacción tiempotratamiento en el análisis de subgrupos del ensayo NINDS; el tratamiento iniciado dentro de los primeros 90 min después del inicio de los síntomas se asoció a una evolución favorable (OR: 2,11; IC 95\%:1,33-3,55); en tanto, para pacientes tratados entre 90 y 180 min después del debut la OR fue de 1,69 (IC 95\%: 1,09$2,62) \cdot{ }^{20}$

Un análisis combinado de seis ECAs con rtPA mostró que el beneficio es mayor mientras más temprano recibe el paciente la terapia trombolítica. ${ }^{21}$ El mejor resultado se observa en pacientes tratados dentro de las primeras $3 \mathrm{~h}$ desde el inicio de los síntomas, en tanto, el beneficio parece desaparecer a las 4,5 h.

La preocupación principal respecto al tratamiento trombolítico es la HIC secundaria al tratamiento. Actualmente está bien definido que el riesgo de HIC es proporcional al grado en que es violado el protocolo utilizado por estudio NINDS para definir el paciente elegible. ${ }^{22,23}$ Según datos de este propio estudio, pacientes con puntuación de 20 o más en la NIHSS tienen un riesgo del $17 \%$, comparado con el 3\% de riesgo en aquellos con puntuación menor de 10 en la propia escala. ${ }^{24}$ La presencia de signos tempranos de isquemia en la TC puede también predecir la HIC, cuando estos signos afectan más de $1 / 3$ del territorio de la arteria cerebral media (ACM) en pacientes tratados dentro de las 6 primeras horas; sin embargo, no es inconveniente para la terapia trombolítica dentro de las primeras 3 horas. ${ }^{25,26} \mathrm{La}$ 
tensión arterial (TA) por encima de 180/105, y la hiperglicemia en el debut, se asocian también a un incremento de HIC relacionada con la trombolisis.

Con excepción del estudio NINDS, estos ECAs excluyeron los pacientes mayores de 80 años. Los pacientes ancianos tienen en general un pronóstico más pobre, lo cual parece estar relacionado con la comorbilidad más que con la edad. ${ }^{27,28} \mathrm{La}$ trombolisis en pacientes ancianos, examinada fuera del contexto de ensayos clínicos parece ser segura, sin un incremento del riesgo de HIC. ${ }^{29,30}$ I gualmente, el uso de rtPA ha sido tradicionalmente desestimado en pacientes con déficit neurológico menor o leve; sin embargo, el pronóstico de estos pacientes no es siempre favorable, y el tratamiento trombolítico puede producir una mejor evolución. ${ }^{31}$

Una revisión sistemática sobre el uso de trombolíticos en la fase aguda del ictus isquémico incluyó 18 ECAs con 5727 pacientes tratados con rtPA, urokinasa y estreptokinasa. ${ }^{32} \mathrm{Al}$ analizar el uso de trombolisis en las primeras $6 \mathrm{~h}$ se obtiene una menor proporción de pacientes con muerte y dependencia en los siguientes tres meses (OR: 0,84; IC95\%: 0,75-0,95), pero mayor riesgo de hemorragia intracraneal sintomática (OR: 3,37; IC95\%: 2,68-4,22), y de muerte en los primeros 10 días (OR: 1,81; IC95\%: 1,46-2,24). Cuando se analizan los tratados dentro de las primeras $3 \mathrm{~h}$ se observa una reducción significativa de muerte y dependencia (OR: 0,66 ; IC95\%: 0,53-0,83) sin efecto sobre la muerte en primeros 10 días (OR: 1,13; IC95\%: 0,86-1,48). Se observó gran heterogeneidad entre los estudios, con variaciones relacionadas con: el agente trombólítico utilizado, el uso de antitrombóticos concomitante a la trombolisis, la gravedad del ictus, y el momento de inicio del tratamiento desde el debut de los síntomas. El grupo tratado con rtPA presentó ligeramente mejores resultados dentro de las primeras $6 \mathrm{~h}$, en tanto el uso de antitrombóticos (aspirina o anticoagulantes) y los ictus más graves (NIHSS > 20 puntos) aumentaron la probabilidad de HIC sintomática.

El estudio fase IV, el Safe Implementation of Thrombolysis in Stroke-Monitoring Study (SITS-MOST), se propuso verificar si el uso de rtPA dentro de las tres primeras horas del inicio de los síntomas es tan seguro como lo notificado en los ECAs, cuando se incorpora en la práctica clínica en un amplio rango de centros. ${ }^{33}$ Este estudio incluyó los países de la Unión Europea más Noruega e Islandia, y participaron hospitales y UI acreditadas; aunque la mitad no tenía experiencia previa con el uso de trombolisis. Tal como en el estudio NINDS se utilizó una dosis de $0,9 \mathrm{mg} / \mathrm{kg}$ y se siguió estrictamente su protocolo. Los resultados se compararon con los datos de los ECAs sobre rtPA de la revisión Cochrane, tanto su brazo de tratamiento (rtPA) como su brazo de control (placebo). Sus resultados muestran que la proporción de casos con HIC y de muertes a los tres meses fue menor que la obtenida de los ECAs realizados con rtPA, en tanto el porcentaje de pacientes con independencia a los tres meses fue mayor ( $54,8 \%$ contra $49 \%$ ). Además, de acuerdo a las diferentes categoría de la escala de Rankin (para valorar independencia a los tres meses), los resultados fueron similares al del grupo de ECAs y muy superiores al grupo placebo de los ECAs con rtPA. Los investigadores del SIST-MOST concluyen que la trombolisis puede considerarse como parte del tratamiento de rutina en pacientes con ictus agudo, elegibles de acuerdo a los criterios del NINDS.

\section{Las oportunidades de tratamiento}

Dado que el éxito de la terapia trombolítica es dependiente del tiempo entre el debut de los síntomas y el momento del tratamiento, la trombolisis se ha convertido en el principal objetivo de la organización de los servicios prehospitalarios, de emergencia y hospitalarios. Ante este reto, se puede 
preguntar... ¿Cómo hacer más efectivo el sistema de salud en aras de aumentar el número de pacientes elegibles para la trombolisis? La respuesta está nuevamente en los resultados científicos obtenidos en las investigaciones sobre tres aspectos básicos para reducir el tiempo entre el inicio de los síntomas y la llegada a la unidad de atención: 1 . Identificación del ictus, 2. Llegada rápido al hospital adecuado, y 3. Evaluación precisa y rápida en el departamento de emergencias.

Identificación del ictus

Según estudios realizados en sociedades desarrolladas, más del $25 \%$ de los pacientes con ictus llegan al departamento de emergencias dentro de las primeras $3 \mathrm{~h}$ tras el debut. ${ }^{34,35}$ Varios estudios han demostrado que la falta de percepción de urgencia sobre el ictus es uno de los factores asociados a la demora en la llegada al hospital, ${ }^{36,37}$ sólo aquellos pacientes con ictus graves identificaron su problema como una urgencia y acudieron rápidamente por atención médica. ${ }^{38,39}$

Varios estudios han demostrado la eficacia de programas de educación a la población y a los profesionales para incrementar el número de pacientes que llega al hospital en tiempo para la aplicación de este tratamiento. ${ }^{40-42}$ Un estudio cuasiexperimental, de intervención comunitaria, desarrollado en tres fases, ha demostrado la eficacia de un programa agresivo de intervención que involucra a la población y a los profesionales; este aumentó en 10 veces el porcentaje de pacientes que recibe tratamiento trombolítico. ${ }^{43,44}$

La intervención sobre la población incluyó: 1. Habilidades para identificar los síntomas de un ictus. 2. Reconocimiento de estos síntomas como una urgencia, lo que implica la búsqueda rápida de atención médica. Las estrategias utilizadas fueron: anuncios públicos por radio, televisión y prensa plana, entrenamiento de activistas de la comunidad (para la difusión de boca en boca), colocación de carteles llamativos en farmacias, hospitales, policlínicos y grandes centros de trabajo. Los términos utilizados fueron los de "ataque cerebral" para referirse al ictus y "cada minuto cuenta" para ilustrar su carácter de urgencia. ${ }^{43,44}$ Por otra parte, el TIA Working Group, reunido para redefinir el concepto de "ataque isquémico transitorio" ha recomendado el uso del término "ataque cerebral" para el uso de la población, en aras de ofrecer una imagen de emergencia. ${ }^{45}$

La intervención sobre los profesionales de salud (atención primaria, médicos en los departamentos de emergencia, neurólogos y clínicos) incluyó: 1.Habilidades para realizar el diagnóstico diferencial. 2. Esta blecimiento de la hora exacta del debut. 3. Conocimiento del protocolo de administración del trombolítico. Esto se logró mediante cursos cortos de adiestramiento y mediante la definición de protocolos de actuación ante estos pacientes. ${ }^{43,44}$

Llegar rápido al hospital adecuado

Diversos estudios han demostrado que la existencia de un servicio de emergencia médica (SEM) mediante ambulancias se asocia fuertemente con una llegada rápida al centro de atención. En el Genentech Stroke Presentation Survey, el uso de SEM redujo significativamente la demora prehospitalaria, el retraso para realizar TC craneal y la demora total, la cual fue dos veces menor que en pacientes que llegaron por otras

vías. ${ }^{38}$ Otros dos estudios prospectivos demostraron que la transportación mediante SEM es uno de los factores determinantes de la llegada rápida al hospital. ${ }^{37,39}$ En Cuba no existen estudios publicados sobre la efectividad del sistema de recogida de estos pacientes; sin embargo, la práctica cotidiana demuestra que el Sistema 
Integrado de Urgencias Médicas (SIUM) no ha logrado una organización que pueda responder a necesidades que serán mayores con la introducción de la trombolisis cerebral en el país. Una estrategia de recogida mediante códigos telefónicos como el 911 de los EE.UU., es esperable en una organización de este tipo.

Para maximizar estas opciones terapéuticas, deben establecerse guías de tratamiento y protocolos de traslado para asegurar una transición ordenada del paciente desde el ambiente prehospitalario hasta el hospital. ${ }^{13,46,47}$ Los objetivos de la fase de SEM en el cuidado de pacientes con ictus son los siguientes:

1.Estabilización $(A B C)$. 2. Identificación rápida del ictus como causa de los hallazgos del paciente. 3. Eliminación de enfermedades que pueden simular un ictus. 4. Transportación rápida al departamento de emergencias (DE) de un centro apropiado. 5. Aviso al centro que recibirá al paciente (código ictus). Estos pasos son especialmente críticos para el uso de terapéuticas tiempo-dependientes como la trombolisis.

A partir de algunas investigaciones, el NINDS ha recomendado el uso de un examen rápido que incluya tres aspectos clínicos: 1. Paresia facial. 2. Caída de un miembro. 3. Trastorno para el habla; siempre que no exista afectación importante de la conciencia (coma) y fuera del contexto de trauma craneal. ${ }^{48}$ Escalas preclínicas, como la de Cincinnati, han demostrado que la identificación del paciente con ictus en la fase pre-hospitalaria puede lograr una sensibilidad de $100 \%$ y una especificidad de $88 \% .{ }^{49,50}$ En un estudio sobre 821 pacientes consecutivos diagnosticados como "ictus probable" bajo estos criterios, sólo el $13 \%$ tenía otras enfermedades simuladoras de un ictus. ${ }^{51}$

En relación con la notificación al centro de traslado, algunas investigaciones han demostrado que el establecimiento de un sistema de código de prioridad para el ictus entre la atención pre-hospitalaria y la unidad donde será recibido el paciente, disminuye significativamente la demora intrahospitalaria, ${ }^{50,52}$ y que puede aumentar hasta $1 / 3$ el número de pacientes elegibles sometidos a trombolisis. ${ }^{47}$ Una línea telefónica directa abierta durante las $24 \mathrm{~h}$ con el especialista de guardia en la UI, parece ser la mejor estrategia. ${ }^{47}$

Evaluación precisa y rápida en el departamento de emergencia

Los centros que reciben y atienden pacientes con ictus deben contar con protocolos que minimicen el tiempo entre la llegada del pacientes y el inicio del tratamiento, lo cual va dirigido a identificar rápidamente los pacientes tributarios de trombolisis cerebral. El tiempo recomendado por el NINDS no será mayor de $60 \mathrm{~min} .{ }^{52}$ Este tipo de protocolo ha logrado aumentar significativamente el número de pacientes sometidos a trombolisis en base a una reducción del tiempo entre la llegada y el tratamiento, un estudio prospectivo realizado en Canadá mostró una reducción del tiempo puerta-TC cráneo en 11 min, y una reducción del tiempo puerta-aguja (tratamiento) en $18 \mathrm{~min}^{53}$

El tratamiento trombolítico será más efectivo si se realiza dentro de una UI. La atención en este tipo de unidades incrementa la utilización de trombolíticos en siete veces. ${ }^{54}$ Además, las UI garantizan un mejor cumplimiento del protocolo y con ello menor riesgo de complicaciones hemorrágicas con el uso del trombolítico. De manera que la unión de ambas estrategias potencia las posibilidades de éxito terapéutico en pacientes con ictus.

\section{Recomendaciones}


De los resultados antes discutidos pueden derivarse las siguientes recomendaciones a las autoridades de salud:

1. Crear una red de unidades de ictus con la dotación necesaria para la atención de los pacientes con accidente vascular cerebral agudo (ictus).

2. Crear un comité de acreditación de unidades de ictus y centros para la atención a estos pacientes. Este comité se regirá por requisitos predefinidos, y debe ser seleccionado por los organismos rectores de la neurología en el país.

3. Introducir la trombolisis cerebral con el factor tisular activador del plasminógeno (rtPA) como un tratamiento de primera línea que puede realizarse en centros y unidades de ictus acreditadas para ello.

4. Realizar campañas educativas de intervención en poblaciones y/o comunidades para: a. Lograr en la población habilidades para identificar precozmente el ictus, b. Convencer a la población sobre la necesidad de acudir al médico de forma inmediata.

5. Difundir el término "ataque cerebral" y la frase "cada minuto cuenta" en programas de intervención poblacionales o comunitarios.

6. Adiestrar al personal que se desempeña en los departamentos o servicios de emergencia (cuerpos de guardia) de policlínicos y hospitales en: a. Habilidades para reconocer el ictus. b. Habilidades para realizar un adecuado diagnóstico diferencial. c. Establecimiento de la hora de debut de los síntomas. d. Conocimiento del protocolo de actuación ante un ictus.

7. Desarrollar un sistema adecuado de recuperación y traslado de los pacientes con ictus hacia los centros de atención mediante ambulancias, con una prioridad similar al infarto agudo del miocardio (IMA) y el politraumatismo.

8. Los centros que atienden pacientes con ictus deben establecer protocolos bien definidos para la atención urgente que garantice la llegada del paciente a la unidad específica de atención en el menor tiempo posible.

9. Establecer indicadores sobre la calidad del funcionamiento de los protocolos para la atención al paciente con ictus en función de los tiempos de demora: a. tiempo puerta-evaluación, b. tiempo puerta-TC craneal, c. tiempo puerta-tratamiento específico.

\section{REFERENCI AS BI BLI OGRÁFICAS}

1. Alvarez-Sabín J, de Leciñana MA, Gállego J, Gil-Peralta A. Plan de atención sanitaria al ictus. Neurología. 2006;21(10):717-26.

2. Stroke Unit Trialists' Collaboration. Atención hospitalaria organizada (unidad de accidentes cerebrovasculares) para el accidente cerebrovascular (Cochrane Review). In: Oxford, Update Software: The Cochrane Library; 2006. [The Cochrane Library, Issue 1, 2006].

3. Kalra L, Evans A, Perez I, Knapp M. A randomised controlled comparison of alternative strategies in stroke care. Health Technol Assess. 2005;9(18). 
4. Rudd AG, Hoffman A, Irwin P, Lowe D, et al. Stroke Unit Care and Outcome: results from the 2001 National Sentinel Audit of Stroke (England, Wales, and Northern I reland). Stroke. 2005; 36: 103-6.

5. Sulter G, Elting JW, Langedijk M, Maurits NM. Admiting acute ischemic stroke to a care monitoring unit versus a conventional stroke unit: a randomized pilot study. Stroke. 2003; 34:101-4.

6. Cavallini A, Micieli G, Marcheselli S, Quaglini S. Role of monitoring in management of acute ischemic stroke patients. Stroke. 2003; 34:2599-603.

7. Candelise L, Gattinoni M, Bersano A, Micieli G. PROSIT Study Group. Stroke-unit care for acute stroke patients: an observational follow-up study. Lancet. 2007; 369(9558): 299-305.

8. Dodel RC, Haacke C, Zamzow K, Paweilik S. Resource utilization and costs of stroke unit care in Germany. Value Health. 2004; 7(2): 144-52.

9. Epifanov $Y$, Dodel R, Haacke C, Schaeg M. Costs of acute stroke care on regular neurological wards: a comparison with stroke unit setting. Health Policy. 2007; 81(2-3): 339-49.

10. Launois R, Giroud M, Mégnigbeto AC, Le Lay K, Présenté G. Estimating the costeffectiveness of stroke units in France compared with conventional care. Stroke. 2004; 35: 770-5.

11. Seenan $\mathrm{P}$, Long $\mathrm{M}$, Langhorne $\mathrm{P}$. Stroke units in their natural habitat: systematic review of observational studies. Stroke. 2007; 38: 1886-92.

12. Alberts MJ, Hademenos G, Latchaw RE, Jagoda A. Brain Attack Coalition. Recommendations for the establishment of primary stroke centers. JAMA. 2000; 283: 3102-9.

13. Alberts MJ, Latchaw RE, Selman WR, Shephard T. Brain Attack Coalition. Recommendations for comprehensive stroke centers: a consensus statement from the Brain Attack Coalition. Stroke. 2005; 36: 1597-616.

14. The National Institute of Neurological Disorders and Stroke rt-PA Stroke Study Group. Tissue plasminogen activator for acute ischaemic stroke. N Engl J Med. 1995; 333: 1581-7.

15. Kwiatkowski TG, Libman RB, Frankel M, Tilley BC. Effects of tissue plasminogen activator for acute ischemic stroke at one year. National Institute of Neurological Disorders and Stroke Recombinant Tissue Plasminogen Activator Stroke Study Group. N Engl J Med. 1999;340:1781-7.

16. Demaerschalk BM, Yip TR. Economic benefit of increasing utilization of intravenous tissue plasminogen activator for acute ischemic stroke in the United States. Stroke. 2005; 36:2500-3.

17. Hacke W, Kaste M, Fieschi C. For the second european-australasian acute stroke study investigators. Randomised double blind placebo-controlled trial of thrombolytic therapy with intravenous alteplase in acute ischaemic stroke (ECASS II). Lancet. 1998; 352: 1245-51. 
18. Clark WM, Albers GW, Madden KP, Hamilton S. For the thromblytic therapy in acute ischemic stroke study investigators. The rtPA (alteplase) 0- to 6-hour acute stroke trial, part A (A0276g): results of a double-blind, placebo-controlled, multicenter study. Stroke. 2000;31:811-6.

19. Clark WM, Wissman S, Albers GW, Jhamandas JH. Recombinant tissue-type plasminogen activator (Alteplase) for ischemic stroke 3 to 5 hours after symptom onset. The ATLANTIS Study: a randomized controlled trial. JAMA. 1999;282:201926.

20. Marler JR, Tilley BC, Lu M, Brott TG. Early stroke treatment associated with better outcome: the NINDS rt-PA stroke study. Neurology. 2000;55: 1649-55.

21. Hacke W, Donnan G, Fieschi C, Kaste M. ATLANTIS Trials Investigators, ECASS Trials Investigators, NI NDS rt-PA Study Group Investigators. Association of outcome with early stroke treatment: pooled analysis of ATLANTIS, ECASS, and NINDS rt-PA stroke trials. Lancet. 2004;363: 768-74.

22. Katzan IL, Hammer MD, Hixson ED, Furlan AJ. Cleveland clinic health system stroke quality improvement team. Utilization of intravenous tissue plasminogen activator for acute ischemic stroke. Arch Neurol. 2004;61:346-50.

23. Graham GD. Tissue plasminogen activator for acute ischemic stroke in clinical practice: a meta-analysis of safety data. Stroke. 2003;34:2847-50.

24. The NINDS t-PA Stroke Study Group. Intracerebral hemorrhage after intravenous t-PA therapy for ischemic stroke. Stroke. 1997;28:2109-18.

25. Patel SC, Levine SR, Tilley BC, Grotta JC. Lack of clinical significance of early ischemic changes on computed tomography in acute stroke. JAMA. 2001;286:28308.

26. Larrue V, von Kummer R, del Zoppo G, Bluhmki E. Hemorrhagic transformation in acute ischemic stroke. Potential contributing factors in the European Cooperative Acute Stroke Study. Stroke. 1997; 28:957-60.

27. Engelter ST, Fluri F, Buitrago-Tellez C, March S. Life-threatening orolingual angioedema during thrombolysis in acute ischemic stroke. J Neurol 2005; 252:116770.

28. Sylaja PN, Cote R, Buchan AM, Hill MD. Thrombolysis in patients older than 80 years with acute ischaemic stroke: Canadian Alteplase for Stroke Effectiveness Study. J Neurol Neurosurg Psychiatry. 2006; 77:826-9.

29. Tanne D, Kasner SE, Demchuk AM, Karen-Moraq N. Markers of increased risk of intracerebral hemorrhage after intravenous recombinant tissue plasminogen activator therapy for acute ischemic stroke in clinical practice: the Multicenter rt-PA Stroke Survey. Circulation. 2002; 105: 1679-85.

30. van Oostenbrugge RJ, Hupperts RM, Lodder J. Thrombolysis for acute stroke with special emphasis on the very old: experience from a single Dutch centre. J Neurol Neurosurg Psychiatry. 2006; 77(3):375-7. 
31. Smith EE, Abdullah AR, Petkovska I, Rosenthal E. Poor outcomes in patients who do not receive intravenous tissue plasminogen activator because of mild or improving ischemic stroke. Stroke. 2005; 36:2497-9.

32. Wardlaw JM, Zoppo G, Yamaguchi T, Berge E. Thrombolysis for acute ischaemic stroke. Cochrane Database Syst Rev. 2003; (3): CD000213.

33. Wahlgren N, Ahmed N, Dávalos A, Ford GA. For the SITS-MOST investigators. Thrombolysis with alteplase for acute ischaemic stroke in the Safe Implementation of Thrombolysis in Stroke-Monitoring Study (SITS-MOST): an observational study. Lancet. 2007;369:275-82.

34. Barber PA, Zhang J, Demchuk AM, Hill MD. Why are stroke patients excluded from TPA therapy? An analysis of patient eligibility. Neurology. 2001;56:1015-20.

35. Reeves MJ, Arora S, Broderick JP, Frankel M. Acute stroke care in the US: results from 4 pilot prototypes of the Paul Coverdell National Acute Stroke Registry. Stroke. 2005; 36: 1232-40.

36. Harraf F, Sharma AK, Brown MM, Lees KR. For the Acute Stroke Intervention Study Group. A multicentre observational study of presentation and early assessment of acute stroke. BMJ . 2002; 325 (6): 1-5.

37. Rossnagel K, J ungehulsing GJ, Nolte $\mathrm{CH}$, Muller-Nordhorn J. Out-of-hospital delays in patients with acute stroke. Ann Emerg Med. 2004;44:476-83.

38. Morris DL, Rosamond W, Madden K, Schultz C. Prehospital and emergency department delays after acute stroke: the Genentech Stroke Presentation Survey. Stroke. 2000;31:2585-90.

39. Lacy CR, Suh DC, Bueno M, Kostis JB. Delay in presentation and evaluation for acute stroke: Stroke Time Registry for Outcomes Knowledge and Epidemiology (S.T.R.O.K.E.). Stroke. 2001;32:63-9.

40. Alberts MJ, Perry A, Dawson DV, Bertels C. Effects of public and professional education on reducing the delay in presentation and referral of stroke patients. Stroke. 1992; 23:352-6.

41. Pancioli AM, Broderick J, Kothari R, Brott T. Public perception of stroke warning signs and knowledge of potential risk factors. J AMA. 1998;279: 1288-92.

42. Segura T, Vega G, Lopez S, Rubio F. Public perception of stroke in Spain. Cerebrovasc Dis. 2003; 16:21-6.

43. Morgenstern LB, Bartholomew LK, Grotta JC, Staub L. Sustained benefit of a community and professional intervention to increase acute stroke therapy. Arch Intern Med. 2003; 163: 2198-202.

44. Morgenstern LB, Staub L, Chan W, Wein TH. Improving delivery of acute stroke therapy: the TLL Temple Foundation Stroke Project. Stroke. 2002;33:160-6.

45. Easton JD, Albers GW, Caplan LR, Saver JL. For the TIA Working Group. Reconsideration of TIA terminology and definitions. Neurology. 2004;62:S29-34. 
46. Belvis R, Cocho D, Marti-Fabregas J, Pagonabarraga J. Benefits of a prehospital stroke code system: feasibility and efficacy in the first year of clinical practice in Barcelona, Spain. Cerebrovasc Dis. 2005; 19: 96-101.

47. Goldstein LB, Simel DL. Is This Patient Having a Stroke? J AMA. 2005; 293(19): 2391-402.

48. Kothari R, Barsan W, Brott T, Broderick J. Frequency and accuracy of prehospital diagnosis of acute stroke. Stroke. 1995;26:937-41.

49. Norris JW, Hachinski VC. Misdiagnosis of stroke. Lancet. 1982; 1:328-31.

50. Kothari R, Hall K, Brott T, Broderick J. Early stroke recognition: developing an out-of-hospital NIH Stroke Scale. Acad Emerg Med. 1997; 4:986-90.

51. Bray JE, Martin J, Cooper G, Barger B. An interventional study to improve paramedic diagnosis of stroke. Prehosp Emerg Care. 2005; 9:297-302.

52. 2005 American Heart Association Guidelines for Cardiopulmonary Resuscitation and Emergency Cardiovascular Care. Circulation. 2005; 112 (suppl I): IV1-IV203.

53. Mehdiratta M, Woolfenden AR, Chapman KM, Johnston DC. Reduction in IV t-PA door to needle times using an Acute Stroke Triage Pathway. Can J Neurol Sci. 2006; 33(2): 214-6.

54. Lattimore SU,Chalela J, Davis L,DeGraba T. NINDS Suburban Hospital Stroke Center.Impact of establishing a primary stroke center at a community hospital on the use of thrombolytic therapy: the NINDS Suburban Hospital Stroke Center experience. Stroke.2003;34(6):55-7.

Recibido: 6 de julio de 2007.

Aprobado: 2 de noviembre de 2007.

Otman Fernández Concepción. Loma Edificio MINAZ apto. 5L. Plaza. La

Habana10600, Cuba. Teléf.: 8818019, E-mail: otmanfc@infomed.sld.cu 